\title{
Concatenated convolutional Codes: Analysis of control properties under linear systems theory point of view
}

\author{
M. I. García-Planas*, El M. Soudi ${ }^{\dagger}$, L. E. Um ${ }^{\ddagger}$ \\ * Matemàtica Aplicada I, \\ Universitat Politècnica de Catalunya, C. Minería 647, Esc. C, 1-3, 08038 Barcelona, Spain \\ (e-mail: maria.isabel.garcia@upc.edu) \\ $\dagger$ Laboratoire de Recherche Mathématiques, Informatique et Applications \\ Université Mohammed V, Avenue des Nations Unies, Agdal, BP: 554, Rabat, Morocco \\ (e-mail: emsouidi@yahoo.com) \\ $\dagger$ Université Mohammed V, Rabat, Morocco \\ (e-mail: laurainlee@yahoo.fr)
}

\begin{abstract}
In this paper we consider two models of concatenated convolutional codes from the perspective of linear systems theory. We present an input-state-output representation of these models and we study the conditions for control properties as controllability, observability as well as output observability.
\end{abstract}

Index Terms-Convolutional codes, linear systems, output-observability

\section{Introduction}

In coding theory, concatenated codes form a class of error-correcting codes that are obtained by combining an inner code and an outer code. They were conceived in 1966 by Dave Forney as a solution to the problem of finding a code that has both exponentially decreasing error probability with increasing block length and polynomial-time decoding complexity. Concatenated codes became widely used in space communications in the 1970s. More Concretely t he concatenation of convolutional codes is used for deep-space transmissions, including also bar codes, the ISBN code for books, and the ones used for credit cards or identity cards. It is well known that the code with the correction capacities that best fit the reliability of the physical devices is used in each instance of information processing. One of these classes of codes are Turbo Codes (which combine two convolutional codes), they are used in mobile telecommunications standards and its variation for internet access. In this paper we study two kinds of concatenated convolutional codes (serial and parallel) using linear systems theory.

It is well known that convolutional codes can be described using a quite more general theory, the linear systems theory over finite fields (see [19], [20], [21] for example).

Following the work initiated in [14], the aim of this article is to give a input-output representation of a concatenated (serial and parallel) convolutional, and deduce conditions for control properties as controllability, observability as well output observability. The control properties are relied to the minimality of strict equivalent encoders.

\section{Preliminaries}

Throughout the paper, we denote by $\mathbb{F}=G F(q)$ the Galois field of $q$ elements and $\overline{\mathbb{F}}$ the algebraic closure of $\mathbb{F}$.

A convolutional code $\mathcal{C}$ of rate $k / n$ and degree $\delta$, 
called an $(n, k, \delta)$-code, can be given by the inputstate-output representation (see [16], [19], [21])

$$
\begin{aligned}
x(t+1) & =A x(t)+B u(t), \\
y(t) & =C x(t)+D u(t), \\
v(t) & =\left(\begin{array}{l}
y(t) \\
u(t)
\end{array}\right), \quad x(0)=0,
\end{aligned}
$$

where for each instant $t, x(t) \in \mathbb{F}^{\delta}$ is the state vector, $u(t) \in \mathbb{F}^{k}$ is the information vector, $y(t) \in \mathbb{F}^{n-k}$ is the parity vector and $v(t)$ is a codeword of $\mathcal{C}$. In that case, $\mathcal{C}$ is said to be generated by the quadruple of matrices $(A, B, C, D)$ and we will denote it by $\mathcal{C}(A, B, C, D)$.

Here $A, B, C$ and $D$ are matrices of sizes $\delta \times \delta$, $\delta \times k,(n-k) \times \delta$ and $(n-k) \times k$, respectively, that is, $(A, B, C, D)$ is a minimal representation and it is characterized through the condition that the pair $(A, B)$ is controllable, i.e.,

$$
\operatorname{rank}\left(\begin{array}{llll}
B & A B & \ldots & A^{\delta-1} B
\end{array}\right)=\delta
$$

or equivalently (see [17]),

$$
\operatorname{rank}(z I-A \quad B)=\delta, \forall z \in \mathbb{F} .
$$

A pair $(A, C)$ is said to be an observable pair if $\left(A^{t}, C^{t}\right)$ is a controllable pair, i.e.,

$$
\operatorname{rank}\left(\begin{array}{c}
C \\
C A \\
\vdots \\
C A^{\delta-1}
\end{array}\right)=\delta
$$

or equivalently (see [17]),

$$
\operatorname{rank}\left(\begin{array}{c}
z I-A \\
C
\end{array}\right)=\delta, \forall z \in \mathbb{F} .
$$

We define a convolutional code $\mathcal{C}(A, B, C, D)$ to be observable if one and therefore every encoder $G(z)$ is right prime. If $G(z)$ is an encoder of an observable convolutional code, then $G(z)$ is necessarily a non-catastrophic encoder, which means that any finite sequence of information, encoded by $G(z)$ will remain finite. The following result shows that if $(A, B)$ is controllable, then the observability of the pair $(A, C)$ ensures that the linear system (1) describes a non-catastrophic convolutional encoder.

Lemma 2.1 (Lemma 2.11 of [21]): Assume that the matrices $(A, B)$ form a controllable pair. The convolutional code $\mathcal{C}(A, B, C, D)$ defined through
(1) represents an observable convolutional code if and only if $(A, C)$ forms an observable pair.

Ch. Fragouli and R. D. Wesel [10] give the following definition of output observability.

Definition 2.1: The system $(A, B, C, D)$ is said to be output observable if the state sequence $\left\{x_{0}, x_{1}, \ldots, x_{n-1}\right\}$ is uniquely determined by the knowledge of the output sequence $\left\{y_{0}, y_{1}, \ldots, y_{n-1}\right\}$ for a finite number of steps $n-1$.

Taking into account that

$y_{k}=C A^{k} x_{0}+C A^{k-1} B u_{0}+\ldots+C A B u_{k-2}+C B u_{k-1}$,

the output observability is characterized by the following proposition.

Proposition 2.1 ([10]): The system $(A, B, C, D)$ is output observable if and only if the matrix $T \in$ $M_{(n-k) \delta \times(\delta(k+1))}(\mathbb{F})$ defined as

$$
\left(\begin{array}{cccccc}
C & D & & & & \\
C A & C B & D & & & \\
C A^{2} & C A B & C B & D & & \\
\vdots & \vdots & \vdots & \ddots & \ddots & \\
C A^{\delta} & C A^{\delta-1} B & C A^{\delta-2} B & \ldots & C B & D
\end{array}\right)
$$

has full row rank.

If we want to preserve the delay condition we need to add the condition that the matrix $D$ has full rank.

\section{Example 2.1: Given}

$$
\begin{aligned}
& (y(0), y(1), y(2), y(3), y(4), y(5), y(6))= \\
& (1,0,0,0,0,0,1),
\end{aligned}
$$

solving the system

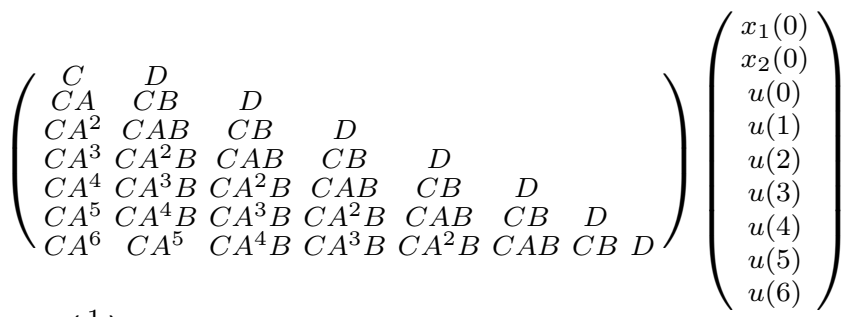

$$
\begin{aligned}
& =\left(\begin{array}{l}
1 \\
0 \\
0 \\
0 \\
0 \\
0 \\
1
\end{array}\right) \text {. }
\end{aligned}
$$


Concretely:

$$
\left(\begin{array}{lllllllll}
1 & 0 & 1 & 0 & 0 & 0 & 0 & 0 & 0 \\
0 & 1 & 1 & 1 & 0 & 0 & 0 & 0 & 0 \\
1 & 0 & 0 & 1 & 1 & 0 & 0 & 0 & 0 \\
0 & 1 & 1 & 0 & 1 & 1 & 0 & 0 & 0 \\
1 & 0 & 0 & 1 & 0 & 1 & 1 & 0 & 0 \\
0 & 1 & 1 & 0 & 1 & 0 & 1 & 1 & 0 \\
1 & 0 & 0 & 1 & 0 & 1 & 0 & 1 & 1
\end{array}\right)\left(\begin{array}{c}
x_{1}(0) \\
x_{2}(0) \\
u(0) \\
u(1) \\
u(2) \\
u(3) \\
u(4) \\
u(5) \\
u(6)
\end{array}\right)=\left(\begin{array}{l}
1 \\
0 \\
0 \\
0 \\
0 \\
0 \\
1
\end{array}\right) .
$$

The solution is

$$
\begin{aligned}
x_{1} & =0 u(5)+1 u(6)+1 \\
x_{2} & =1 u(5)+0 u(6)+1 \\
u(0) & =0 u(5)+1 u(6)+0 \\
u(1) & =1 u(5)+1 u(6)+1 \\
u(2) & =1 u(5)+0 u(6)+0 \\
u(3) & =0 u(5)+1 u(6)+1 \\
u(4) & =1 u(5)+1 u(6)+1
\end{aligned}
$$

that for $x_{1}(0)=x_{2}(0)=0$ we obtain:

$$
\begin{aligned}
& (u(0), u(1), u(2), u(3), u(4), u(5), u(6))= \\
& (1,1,1,0,1,1,1)= \\
& 1+s+s^{2}+s^{4}+s^{5}+s^{6}= \\
& Q(s) m(s)=\left(1+s^{2}\right) m(s) .
\end{aligned}
$$

Then $m(s)=1+s+s^{3}+s^{4}$.

Finally, in terms of the input-state-output representation (1), the free distance of a convolutional code $\mathcal{C}$, that is, the minimum Hamming distances between any two code sequences of $\mathcal{C}$, can be characterized as (see [16])

$$
d_{\text {free }}(\mathcal{C})=\lim _{j \rightarrow \infty} d_{j}^{c}(\mathcal{C})
$$

where

$$
d_{j}^{c}(\mathcal{C})=\min _{u(0) \neq 0}\left\{\sum_{t=0}^{j} w t(u(t))+\sum_{t=0}^{j} w t(y(t))\right\}
$$

is the $j$-th column distance of the convolutional code $\mathcal{C}$, for $j=0,1,2, \ldots$.

\section{Concatenation}

In this section we introduce the following models of concatenation of two convolutional codes.

The first model considered is the following.

Let $\mathcal{C}_{\mathbf{O}}\left(A_{1}, B_{1}, C_{1}, D_{1}\right)$ and $\mathcal{C}_{\mathbf{i}}\left(A_{2}, B_{2}, C_{2}, D_{2}\right)$ be convolutional codes, called outer code, and inner code respectively. Let $x_{1}(t), u_{1}(t)$, and $y_{(1)}(t)$ be the state vector, the information vector and the parity vector of $\mathcal{C}_{\mathbf{O}}\left(A_{1}, B_{1}, C_{1}, D_{1}\right)$, and let $x_{2}(t)$, $u_{2}(t)$, and $y_{2}(t)$ be the state vector, the information vector and the parity vector of $\mathcal{C}_{\mathbf{i}}\left(A_{2}, B_{2}, C_{2}, D_{2}\right)$, respectively.

The outer code $\mathcal{C}_{\mathrm{O}}$ and the inner code $\mathcal{C}_{\mathrm{i}}$ are serialized, one after the other, so that the input information $u_{2}=y_{1}(t)$. Consequently

$$
\begin{aligned}
x_{1}(t+1) & =A_{1} x_{1}(t)+B_{1} u_{1}(t) \\
x_{2}(t+1) & =A_{2} x_{2}(t)+B_{2} C_{1} x_{1}(t)+B_{2} D_{1} u_{1}(t) \\
y_{2}(t) & =C_{2} x_{2}(t)+D_{2} C_{1} x_{1}(t)+D_{2} D_{1} u_{1}(t)
\end{aligned}
$$

That is to say the concatenated code is $\mathcal{C}(A, B, C, D)$ with

$$
\begin{gathered}
A=\left(\begin{array}{cc}
A_{1} & 0 \\
B_{2} C_{1} & A_{2}
\end{array}\right), B=\left(\begin{array}{c}
B_{1} \\
B_{2} D_{1}
\end{array}\right), \\
C=\left(\begin{array}{ll}
D_{2} C_{1} & C_{2}
\end{array}\right), D=D_{2} D_{1} .
\end{gathered}
$$

If $\mathcal{C}_{0}\left(A_{1}, B_{1}, C_{1}, D_{1}\right)$ is a $\left(m, k, \delta_{1}\right)$-code and $\mathcal{C}_{i}\left(A_{2}, B_{2}, C_{2}, D_{2}\right)$ is a $\left(n, m-k, \delta_{2}\right)$-code, then $\mathcal{C}(A, B, D)$ is a $\left(n-m+2 k, k, \delta_{1}+\delta_{2}\right)$-code.

The second model presented is the parallel concatenation. Let $\mathcal{C}_{1}\left(A_{1}, B_{1}, C_{1}, D_{1}\right)$ and $\mathcal{C}_{2}\left(A_{2}, B_{2}, C_{2}, D_{2}\right)$ be convolutional codes. Let $x_{1}(t), u_{1}(t)$, and $y^{(1)}(t)$ be the state vector, the information vector and the parity vector of $\mathcal{C}_{1}\left(A_{1}, B_{1}, C_{1}, D_{1}\right)$, and let $x_{2}(t), u_{2}(t)$, and $y_{2}(t)$ be the state vector, the information vector and the parity vector of $\mathcal{C}_{2}\left(A_{2}, B_{2}, C_{2}, D_{2}\right)$, respectively.

Both codes are concatenated in a parallel form, so that the input information $u_{2}(t)=u_{1}(t)=u(t)$ and the final parity vector $y(t)=y_{1}(t)+y_{2}(t)$. Consequently

$$
\begin{aligned}
x_{1} & =A_{1} x_{1}(t)+B_{1} u(t) \\
x_{2} & =A_{2} x_{2}(t)+B_{2} u(t) \\
y(t) & =C_{1} x_{1}(t)+C_{2} x_{2}(t)+\left(D_{1}+D_{2}\right) u(t) \\
& A=\left(\begin{array}{cc}
A_{1} & 0 \\
0 & A_{2}
\end{array}\right), B=\left(\begin{array}{l}
B_{1} \\
B_{2}
\end{array}\right) \\
& C=\left(\begin{array}{ll}
C_{1} & C_{2}
\end{array}\right), D=D_{1}+D_{2} .
\end{aligned}
$$

If $\mathcal{C}_{1}\left(A_{1}, B_{1}, C_{1}, D_{1}\right)$ is a $\left(n, k, \delta_{1}\right)$-code and $\mathcal{C}_{2}\left(A_{2}, B_{2}, C_{2}, D_{2}\right)$ is a $\left(n, k, \delta_{2}\right)$-code, then $\mathcal{C}(A, B, C, D)$ is a $\left(n, k, \delta_{1}+\delta_{2}\right)$-code. 


\section{Control properties}

In this section, we establish conditions on the linear systems with matrices $\left(A_{i}, B_{i}, C_{i}, D_{i}\right)$ of the inner convolutional code $\mathcal{C}_{i}$ in order to obtain an observable convolutional code with a minimal representation from the different models of concatenation introduced in Section 3, that is, a representation with the pair $(A, B)$ controllable and the pair $(A, C)$ observable.

\subsection{Serial concatenated case}

Following Hautus theorem

a) the concatenated system $(A, B, C, D)$ is controllable if and only if the matrix

$$
\left(\begin{array}{ccc}
z I_{\delta_{1}}-A_{1} & 0 & B_{1} \\
-B_{2} C_{1} & z I_{\delta_{2}}-A_{2} & B_{2} D_{1}
\end{array}\right)
$$

has full row rank $\left(=\delta_{1}+\delta_{2}\right)$, for all $z \in \mathbb{F}$.

b) the concatenated system $(A, B, C, D)$ is observable if and only if the matrix

$$
\left(\begin{array}{cc}
z I_{\delta_{1}}-A_{1} & 0 \\
-B_{2} C_{1} & z I_{\delta_{2}}-A_{2} \\
D_{2} C_{1} & C_{2}
\end{array}\right)
$$

has full column rank $\left(=\delta_{1}+\delta_{2}\right.$, for all $\left.z \in \mathbb{F}\right)$.

Therefore we have the following propositions.

Proposition 4.1: A necessary condition for controllability of concatenated code is that the pair $\left(A_{1}, B_{1}\right)$ be controllable.

Proposition 4.2: A necessary condition for observability of concatenated code is that the pair $\left(A_{2}, C_{2}\right)$ be observable.

Suppose now that $k \geq \delta_{1}+\delta_{2}$, then we get to the proposition

Proposition 4.3: If the matrix $\left(\begin{array}{c}B_{1} \\ B_{2} D_{1}\end{array}\right)$ has full rank, then the system $(A, B, C, D)$ is controllable.

Corollary 4.1: With the same hypothesis than 4.3, if the matrix $\left(\begin{array}{l}B_{1} \\ B_{2}\end{array}\right)$ has full rank, then the system $(A, B, C, D)$ is controllable. $\delta_{1}+\delta_{2}$, so:

Proof: Because of $k \geq \delta_{1}+\delta_{2}, \operatorname{rank}\left(\begin{array}{l}B_{1} \\ B_{2}\end{array}\right)=$ $\delta_{1}+\delta_{2}=$

$\operatorname{rank}\left(\begin{array}{c}B_{1} \\ B_{2} D_{1}\end{array}\right)=\operatorname{rank}\left(\begin{array}{cc}B_{1} & 0 \\ 0 & B_{2}\end{array}\right)\left(\begin{array}{ll}I_{k} & \\ & D_{1}\end{array}\right) \leq$ $\operatorname{rank}\left(\begin{array}{cc}B_{1} & 0 \\ 0 & B_{2}\end{array}\right)=\operatorname{rank}\left(\begin{array}{l}B_{1} \\ B_{2}\end{array}\right)=\delta_{1}+\delta_{2}$.

Suppose now that $n-k \geq \delta_{1}+\delta_{2}$, the we have the following proposition.

Proposition 4.4: If the matrix $\left(D_{2} C_{1} \quad C_{2}\right)$ has full rank, then the system $(A, B, C, D)$ is observable.

Example 4.1: Over the field $\mathbb{F}=\mathbb{Z}_{5}$, we consider the codes $\mathcal{C}\left(A_{1}, B_{1}, C_{1}, D_{1}\right)$, and $\mathcal{C}\left(A_{2}, B_{2}, C_{2}, D_{2}\right)$, with

$$
A_{1}=(0), B=\left(\begin{array}{ll}
1 & 2
\end{array}\right), C=(4), D=(1
$$

and

$$
\begin{gathered}
A_{2}=\left(\begin{array}{ll}
0 & 4 \\
1 & 0
\end{array}\right), B_{2}=\left(\begin{array}{l}
1 \\
0
\end{array}\right), \\
C_{2}=\left(\begin{array}{ll}
1 & 0
\end{array}\right), D_{2}=(1)
\end{gathered}
$$

The serial concatenated code considered is $(A, B, C, D)$ with

$$
\begin{gathered}
A=\left(\begin{array}{lll}
0 & 0 & 0 \\
4 & 0 & 4 \\
0 & 1 & 0
\end{array}\right), B=\left(\begin{array}{ll}
1 & 2 \\
1 & 3 \\
0 & 0
\end{array}\right) \\
C=\left(\begin{array}{lll}
4 & 1 & 0
\end{array}\right), D=\left(\begin{array}{ll}
1 & 3
\end{array}\right)
\end{gathered}
$$

It is easy to show that the system and $(A, B, C, D)$ is output observable:

And

$\operatorname{rank}\left(\begin{array}{ccccc}C & D & & & \\ C A & C B & D & & \\ C A^{2} & C A B & C B & D & \\ C A^{3} & C A^{2} B & C A B & C B & D\end{array}\right)=$

$\operatorname{rank}\left(\begin{array}{cccccccccccc}4 & 1 & 0 & 1 & 3 & & & & & & \\ 4 & 0 & 4 & 0 & 1 & 1 & 3 & & & & \\ 0 & 4 & 0 & 4 & 3 & 0 & 1 & 1 & 3 & & \\ 1 & 0 & 1 & 4 & 2 & 4 & 3 & 0 & 1 & 1 & 3\end{array}\right)=4$. 


\subsection{Parallel concatenated case}

The controllability matrix of the parallel concatenated code is

$$
\left(\begin{array}{cccc}
B_{1} & A_{1} B_{1} & \ldots & A_{1}^{\delta_{1}+\delta_{2}} B_{1} \\
B_{2} & A_{2} B_{2} & \ldots & A_{1}^{\delta_{1}+\delta_{2}} B_{2}
\end{array}\right)
$$

Proposition 4.5: A necessary condition for controllability of concatenated system is that the pairs $\left(A_{2}, B_{1}\right)$ and $\left(A_{2}, B_{2}\right)$ are controllable

Obviously, this condition it is not sufficient as we can see in the following example:

Example 4.2: Let $A_{i}=(0), B_{i}=(1), C_{i}=(1)$ and $D_{i}=(1)$ for $i=1,2$, the parallel concatenated code is

$$
A=0, B=\left(\begin{array}{l}
1 \\
1
\end{array}\right), C=\left(\begin{array}{ll}
1 & 1
\end{array}\right), D=(2) .
$$

It is obvious that this code is not controllable, but both codes $\left(A_{i}, B_{i}, C_{i}, D_{i}\right)$ are controllable.

The observability matrix of the concatenated code is

$$
\left(\begin{array}{cc}
C_{1} & C_{2} \\
C_{1} A_{1} & C_{2} A_{2} \\
\vdots & \vdots \\
C_{1} A_{1}^{\delta_{1}+\delta_{2}} & C_{2} A_{2}^{\delta_{1}+\delta_{2}}
\end{array}\right)
$$

Proposition 4.6: A necessary condition for observability of concatenated system is that the pairs $\left(A_{1}, C_{1}\right)$ and $\left(A_{2}, C_{2}\right)$ are observable

The same codes in the previous example serve to prove that the converse of this proposition is not true.

Consider now a parallel concatenated code $\mathcal{C}(A, B, C, D)$ obtained from the concatenation of the codes $\mathcal{C}_{1}\left(A_{1}, B_{1}, C_{1}, D_{1}\right)=\mathcal{C}_{2}\left(A_{2}, B_{2}, C_{2}, D_{2}\right)$.

The output observability matrix of this concatenated code is

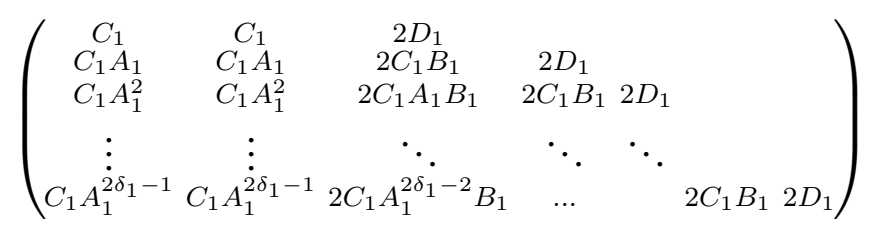

and the rank of this matrix coincides with the rank of

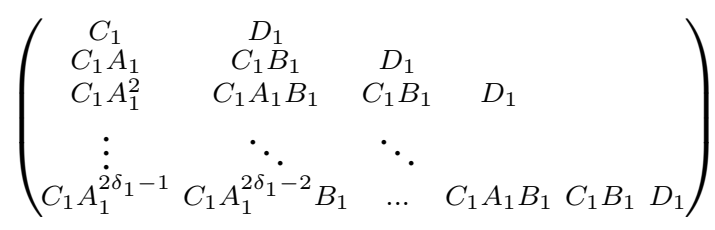

Notice that the submatrix

$$
T_{\delta}=\left(\begin{array}{cccll}
C_{1} & D_{1} & & & \\
C_{1} A_{1} & C_{1} B_{1} & D_{1} & & \\
C_{1} A_{1}^{2} & C_{1} A_{1} B_{1} & C_{1} B_{1} & D_{1} & \\
\vdots & \ddots & \ddots & & \\
C_{1} A_{1}^{\delta_{1}-1} & C_{1} A_{1}^{\delta_{1}-2} B_{1} & \ldots & C_{1} A_{1} B_{1} C_{1} B_{1} D_{1}
\end{array}\right)
$$

on corresponds to the output observability matrix of the $\mathcal{C}_{1}(A, B, C, D)$ code.

Therefore, is having the following proposition.

Proposition 4.7: A necessary condition for output observability of the concatenated code $\mathcal{C}(A, B, C, D)$ is that the code $\mathcal{C}_{1}(A, B, C, D)$ be output observable.

Calling $T_{i}$ the matrix

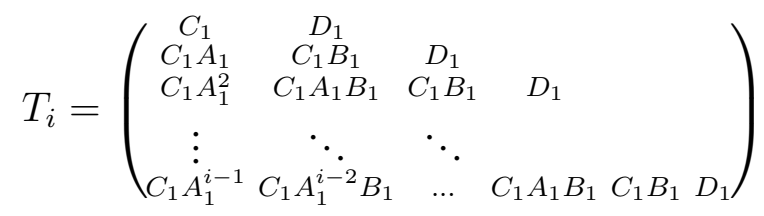

for all $i \geq \delta$, we have the following theorem.

Theorem 4.1: Suppose that the code $\mathcal{C}_{1}(A, B, C, D)$ is output observable. A necessary condition for output observability of the concatenated code $\mathcal{C}(A, B, C, D)$ is that the following eqaulity

$$
\operatorname{rank} T_{\delta+1}-\operatorname{rank} T_{\delta}=n-k
$$

holds.

Proof: Following [13], for all $i \geq \delta$ the relation holds

$$
\operatorname{rank} T_{i+1}-\operatorname{rank} T_{i}=\ell(\text { constant })
$$

Example 4.3: Over the field $\mathbb{F}_{5}$. Let $\mathcal{C}_{1}\left(A_{1}, B_{1}, C_{1}, D_{1}\right) \quad$ and $\quad \mathcal{C}_{2}\left(A_{2}, B_{2}, C_{2}, D_{2}\right) \quad$ be two codes with

$$
\begin{aligned}
& A_{1}=\left(\begin{array}{ll}
0 & 1 \\
0 & 0
\end{array}\right), B_{1}=\left(\begin{array}{ll}
0 & 0 \\
1 & 1
\end{array}\right), \\
& C_{1}=\left(\begin{array}{ll}
1 & 1 \\
1 & 1
\end{array}\right), D_{1}=\left(\begin{array}{ll}
1 & 2 \\
1 & 2
\end{array}\right)
\end{aligned}
$$


and

$$
\begin{aligned}
& A_{2}=\left(\begin{array}{ll}
0 & 0 \\
1 & 0
\end{array}\right), B_{2}=\left(\begin{array}{ll}
1 & 1 \\
0 & 0
\end{array}\right), \\
& C_{2}=\left(\begin{array}{ll}
1 & 1 \\
2 & 2
\end{array}\right), D_{2}=\left(\begin{array}{ll}
1 & 1 \\
2 & 2
\end{array}\right)
\end{aligned}
$$

It is easy to observe that these codes are not output observable:

$$
\begin{aligned}
& \operatorname{rank}\left(\begin{array}{ll}
C_{1} & D_{1}
\end{array}\right)=\operatorname{rank}\left(\begin{array}{cccc}
1 & 1 & 1 & 2 \\
1 & 1 & 1 & 2
\end{array}\right)=1<2, \\
& \operatorname{rank}\left(\begin{array}{ll}
C_{2} & D_{2}
\end{array}\right)=\operatorname{rank}\left(\begin{array}{llll}
1 & 1 & 1 & 1 \\
2 & 2 & 2 & 2
\end{array}\right)=1<2 .
\end{aligned}
$$

Nevertheless, the parallel concatenated system is output observable, for that it suffices to observe that

$$
\operatorname{rank}\left(D_{1}+D_{2}\right)=2
$$

\section{Conclusions}

In this paper a detailed look at the algebraic structure of concatenated (serial and parallel) convolutional codes using techniques of linear systems theory has been made. Conditions for controllability, observability and output-observability has been obtained.

\section{References}

[1] S. Benedetto, D. Divsalar, G. Montorsi, and F. Pollara, Parallel concatenated trellis coded modulation, in Proc. IEEE Int. Conf. Communications, vol. 2, Dallas, TX, June 1996, pp. 974978.

[2] J-J. Climent, V.Herranz, C.Perea, A first approximation of concatenated convolutional codes from linear systems theory viewpoint, Linear Algebra and its Applications 425, pp. 673699, (2007).

[3] J-J. Climent, V.Herranz, C.Perea, Linear system modelization of concatenated block and convolutional codes, Linear Algebra and its Applications, 429, pp. 1191-1212, (2008).

[4] J. Mara Muñoz Porras, J.I. Iglesias Curto, Classification of convolutional codes, Linear Algebra and its Applications vol. 432, Issue 10, pp. 27012725, (2010).

[5] L. Scripcariu, Serial concatenated convolutional codes design and analysis, 8th International Conference on development and applications systems, Suceava Romania, May 25-27, (2006).

[6] W.E. Ryan, "Concatenated Codes and Iterative Decoding," in Wiley Encyclopedia of Telecommunications (J. G. Proakis, ed.) New York : Wiley and Sons, (2003).

[7] W.E. Ryan, Concatenated Convolutional Codes and Iterative Decoding, (2001)

[8] A. Ghrayeb, T. Abualrub, On parallel and serial concatenated convolutional codes over GF(4). The 8th International, Conference on Communication Systems(ICCS), (2002).
[9] F. Garin, G. Como, and F. Fagnani, The performance of serial turbo codes does not concentrate. IEEE Transactions on Information Theory, vol. 58, no. 5, doi: 10.1109/TIT.2012.2184673, May 5, (2012).

[10] Ch. Fragouli, R.D. Wesel, (1999) Convolutional Codes and Matrix Control Theory, Proceedings of the 7th International Conference on Advances in Communications and Control, Athens, Greece.

[11] D. Divsalar and R. J. McEliece, On the design of generalized concatenated coding systems with interleavers, TMO PR 42134, Apr.June (1998).

[12] D. Divsalar and F. Pollara, On the design of turbo codes, TMO PR 42-123, JulySept. (1995).

[13] M- I. García-Planas, M.D. Magret, An alternative System of Structural Invariants for Quadruples of Matrices, Linear Algebra and its Applications 291, (1-3), pp. 83-102 (1999).

[14] M- I. García-Planas, El M. Souidi, L.E. Um, Analysis of control properties of concatenated convolutional codes. Preprint.

[15] M- ${ }^{\mathrm{a}}$ I. García-Planas, El M. Souidi, L.E. Um, Convolutional codes under linear systems point of view. Analysis of outputcontrollability.Wseas Transactions on Mathematics. vol. 11, (4), pp. 324-333, (2012).

[16] R. Hutchinson, J. Rosenthal, R. Smarandache, Convolutional codes with maximum distance profile,Systems Control Lett. 54 (1) (2005) 5363.

[17] M. Hautus, Controllability and observability condition for linear autonomous systems, Proceedings of Nedderlandse Akademie voor Wetenschappen, Series A 72 (1969) 443448.

[18] J.M. Muñoz Porras, J.I. Iglesias Curto, Classification of convolutional codes, Linear Algebra and its Applications 432 (10), (2010), 27012725

[19] J. Rosenthal, J. Schumacher, E.V. York, On behaviors and convolutional codes, IEEE Trans. Inform. Theory 42 (6) (1996) 18811891.

[20] J. Rosenthal, R. Smarandache, Maximum distance separable convolutional codes, Applicable algebra in engineering, Commun. Comput. 10 (1999) 1532.

[21] J. Rosenthal, E.V. York, BCH convolutional codes, IEEE Trans. Inform. Theory 45 (6) (1999) 18331844. 